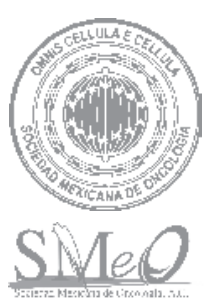

CASO CLÍNICO

\title{
Déficit de magnesio en paciente con quimioterapia-radioterapia por cáncer de cérvix: caso clínico y revisión
}

\section{Miguel Ángel Berenguer-Francés*}

Servicio de Oncología Radioterápica, Hospital Sant Joan d'Alacant, Sant Joan d'Alacant (Alicante), España

Recibido el 1 de octubre de 2016; aceptado el 25 de febrero de 2017

Disponible en Internet el 4 de agosto de 2017

\section{PALABRAS CLAVE \\ Magnesio; \\ Cáncer de cérvix; \\ Radioterapia}

Resumen Introducción: El déficit de magnesio es una afección frecuente en los pacientes oncológicos debido a los esquemas de quimioterapia con cisplatino. La combinación con radioterapia en ciertos tumores en que el tracto gastrointestinal forma parte del campo de tratamiento puede producirlo. Caso clínico: Se presenta el caso de una mujer de 35 años de edad diagnosticada de cáncer de cérvix en tratamiento con quimioterapia y radioterapia concomitante. Debido a la extensión del tumor y a la afectación ganglionar, el campo de tratamiento radioterápico era amplio, con dosis grandes sobre el intestino delgado. Durante el tratamiento, la paciente presentó una diarrea importante debido al déficit de magnesio. Resultados: El estudio de la hipomagnesemia no es habitual en la práctica clínica, pero debe contemplarse en aquellos pacientes que estén en tratamiento con esquemas quimioterápicos que contenga cisplatino y radioterapia pélvica ampliada. Discusión: Conocer esta afección y proporcionar un tratamiento adecuado puede evitar empeoramientos en la calidad de vida de los sujetos afectos, y la posible finalización precoz del tratamiento por mala tolerancia. (creativecommons.org/licenses/by-nc-nd/4.0/).

*E-mail para correspondencia: migberenguer@hotmail.com (M.Á. Berenguer-Francés) 


\section{INTRODUCCIÓN}

El déficit de magnesio es un efecto secundario conocido en pacientes en tratamiento quimioterápico con cisplatino. La hipomagnesemia se define como unos valores del ion en suero inferiores a $1,8 \mathrm{mg} / \mathrm{dl}(1,5 \mathrm{mEq} /)^{1}$, y su relación con el uso de cisplatino es dependiente de la dosis, aumentando su frecuencia con cada ciclo administrado ${ }^{2,3}$. Es uno de los trastornos hidroelectrolíticos más frecuentes, pero sus manifestaciones clínicas son inespecíficas. La clínica suele basarse en astenia, confusión, irritabilidad, convulsiones y alteraciones neuromusculares o cardiacas ${ }^{4}$. Su diagnóstico es difícil, ya que la determinación sérica de magnesio no es habitual. Además, hay una relación estrecha entre las concentraciones de magnesio y las de fósforo y potasio, lo que dificulta poder distinguir si los síntomas clínicos se deben a uno o a otro ion.

El tratamiento del cáncer de cuello uterino se basa, excepto en los estadios muy iniciales, en la combinación de quimioterapia y radioterapia. El uso de cisplatino semanal a dosis de $40 \mathrm{mg} / \mathrm{m}^{2}$, junto con radioterápica pélvica hasta dosis de 46-50 Gy y braquiterapia posterior, es el esquema más empleado. La cirugía exclusiva se reserva para los estadios IA y IB1. Si el estadio del tumor es avanzado, el campo de tratamiento radioterápico puede ser más amplio y llegar, en el límite superior, a la bifurcación de las iliacas o a los ganglios paraaórticos, lo que aumenta considerablemente la posibilidad de una enteritis rádica y, por ende, de problemas de malabsorción.

El tratamiento se basa en la corrección del proceso subyacente, siempre que sea posible. Si el déficit se presenta en las primeras etapas del tratamiento oncológico, pueden administrarse sales de magnesio por vía oral. Si hay compromiso vital, se administran ampollas de sulfato de magnesio al $15 \%$ diluidas en solución salina glucosado al $5 \%$ en 20 minutos, continuando los siguientes días hasta la corrección del déficit. Hay que evitar la administración en bolo por las posibles complicaciones cardiológicas que pueden derivarse de ella $a^{5,6}$.

\section{CASO CLÍNICO}

Se presenta el caso de una mujer de 35 años de edad que desde hacía más de un año presentaba sangrados sin relación con el ciclo menstrual y dolor en el hipogastrio. Ante la clínica descrita, la paciente consulta en ginecología y en la exploración presenta genitales externos y vagina normal, con cuello uterino lateralizado totalmente a la izquierda. Al tacto vaginal se evidencia rigidez en la cara anterolateral izquierda vaginal, en su tercio superior, con crecimiento de una tumoración cervical supravaginal. Al tacto rectal se evidencia parametrio derecho normal y el izquierdo no valorable por el crecimiento de la lesión tumoral. El cérvix estaba en contacto con la cara anterior del recto. En la ecografía ginecológica se aprecia una lesión de $5.2 \times 3.4 \mathrm{~cm}$ en el cérvix, con vascularización abundante e invasión profunda, sin afectación parametrial.

Se realizó el estudio de extensión de la enfermedad, solicitando resonancia magnética pélvica que informa de masa en el cérvix de $5.5 \times 4.5 \times 4 \mathrm{~cm}$, con presencia de adenopatía de $1.2 \mathrm{~cm}$ en la cadena iliaca derecha sin invasión parametrial, vaginal, ureteral, vesical ni rectal. La tomografía computarizada por emisión de positrones evidencia hipercaptación (SUVmax 16.55) en la región cervical, de un diámetro de $5.5 \mathrm{~cm}$, y en la cadena iliaca derecha (SUVmax 12.30). Se realizó biopsia de la lesión con el resultado de carcinoma adenoescamoso de cuello uterino.

Se presentó el caso de esta paciente en el comité multidisciplinario de tumores ginecológicos de nuestro hospital $\mathrm{y}$, ante el resultado anatomopatológico y radiológico, FIGO IIIB, se decidió administrar quimioterapia y radioterapia. La paciente inició tratamiento quimioterápico con cisplatino $\left(40 \mathrm{mg} / \mathrm{m}^{2}\right)$ semanal en combinación con radioterapia. El campo de planificación del tratamiento radioterápico (PTV) llegaba en su límite superior hasta las vértebras L4-L5. Durante el tercer ciclo de quimioterapia, y con una dosis de radioterapia de 22 Gy de los 50 previstos, la paciente presentó náuseas, vómitos y diarrea intensa que no mejoraba a pesar de tomar loperamida y de realizar cambios dieté ticos. Junto a esto, se asociaba una pérdida de 7 kilogramos de peso en 20 días e importante astenia. Además, según referían los familiares, estaba más irascible y con pérdidas de memoria.

Se solicitó hemograma y bioquímica, en la que destacaban unos valores de $\mathrm{K}^{+}$de $3.4 \mathrm{mEq} / \mathrm{l}$ y $\mathrm{Mg}^{++}$de $1.3 \mathrm{mg} / \mathrm{dl}$. Se pautaron comprimidos con $404.85 \mathrm{mg}$ de lactato magnésico cada 8 horas, durante 5 días. La paciente comenzó a notar mejoría en su clínica gastrointestinal y de memoria. Se solicitó nueva analítica después de completar el tratamiento, que mostró unos valores de magnesio de $2.2 \mathrm{mg} / \mathrm{dl}$.

\section{DISCUSIÓN}

La radioterapia pélvica es una de las causas de déficit de magnesio más desconocidas, y esto aumenta si el pacien te precisa tratamiento quimioterápico con cisplatino. Son varios los tumores que requieren campos de tratamiento radioterápico pélvicos extensos que pueden provocar en teritis rádica (cuello uterino, endometrio, próstata, recto y canal anal) y, por tanto, malabsorción de nutrientes. Aunque se conocen las dosis limitantes de los órganos de riesgo, muchas veces estas no pueden cumplirse en su totalidad por la extensión del tumor o la afectación ganglionar.

A pesar de ser un efecto secundario conocido en la literatura (Tabla 1), hay pocos estudios que evalúen el uso profiláctico de magnesio junto con el tratamiento con cisplatino y radioterapia pélvica. Hunter, et al. ${ }^{7}$ estudiaron en más de 200 pacientes el efecto beneficioso de la suplementación con sulfato de magnesio en los pacientes en tratamiento combinado con cisplatino y radioterapia. Además, observaron que los pacientes con diabetes me llitus o con hipertensión eran los que tenían más riesgo de sufrir déficit de magnesio, debido a problemas renales. En su análisis, Evans y Harper ${ }^{8}$ evaluaron una muestra de 28 pacientes con cáncer gastrointestinal tratados con quimioterapia con cisplatino, 5-fluorouracilo y epirubicina, y evidenciaron una mejoría estadísticamente significativa con la suplementación de magnesio después del tercer ciclo de cisplatino. Más recientes son los estudios de Zarif Yeganeh, et al. ${ }^{9} \mathrm{y}$ de Yamamoto, et al. ${ }^{10}$, que también reportan mejoría con el uso profiláctico de suplementos de 
Tabla 1. Estudios que han evaluado el uso de suplementos de magnesio en pacientes con tumores tratados con cisplatino

\begin{tabular}{|c|c|c|c|c|c|}
\hline Autores & Hunter, et al. ${ }^{7}$ & Evans, et al. ${ }^{8}$ & Zarif Yenageh, et al. ${ }^{9}$ & Yamamoto, et al. ${ }^{10}$ & Kidera, et al. ${ }^{11}$ \\
\hline Tipo de estudio & Clínico aleatorizado & Clínico aleatorizado & Clínico aleatorizado & Cohortes & Retrospectivo \\
\hline Año & 2009 & 1995 & 2016 & 2015 & 2014 \\
\hline Tipo de tumor & Cuello uterino & Gastrointestinal & Gastrointestinal & Cuello uterino & Varios \\
\hline $\begin{array}{l}\text { Evaluación uso } \\
\text { de radioterapia }\end{array}$ & Sí & No & No & No & No \\
\hline $\mathrm{N} .^{\circ}$ pacientes & 274 & 28 & 69 & 28 & 401 \\
\hline $\begin{array}{l}\text { Resultado } \\
\text { suplementación }\end{array}$ & Beneficio & Beneficio & Beneficio & Beneficio & Beneficio \\
\hline
\end{tabular}

magnesio en pacientes oncológicos que van a recibir quimioterapia con cisplatino. En el estudio retrospectivo de Kidera, et al. ${ }^{11}$ también se halló un efecto benéfico de los suplementos de magnesio en diferentes tumores sólidos, incluido el cáncer de pulmón.

En la práctica clínica diaria, conocer las concentraciones séricas de magnesio previas a iniciar un tratamiento oncológico con cisplatino y radioterapia pélvica ampliada puede evitar situaciones de déficit que produzcan clínica en los pacientes. En nuestro caso, la calidad de vida de la paciente había empeorado de manera significativa desde el inicio del tratamiento oncológico, y se dudaba de que pudiera completarlo. La administración de suplementos de magnesio propició una mejoría de su clínica, principalmente la gastrointestinal.

\section{CONCLUSIONES}

El déficit de magnesio en los pacientes en tratamiento con cisplatino y radioterapia con campos pélvicos extensos es una situación frecuente en la práctica clínica que, en ocasiones, no es diagnosticada y produce síntomas clínicos gastrointestinales importantes en los pacientes. Realizar de manera sistemática controles de las concentraciones de magnesio y suplementar cuando estas sean bajas es una solución beneficiosa.

\section{AGRADECIMIENTOS}

Al servicio de oncología radioterápica del Hospital de Sant Joan d'Alacant.

\section{CONFLICTO DE INTERESES}

El autor hace constar que no tiene ningún tipo de conflicto de intereses.

\section{BIBLIOGRAFÍA}

1. Ariceta G, Rodríguez-Soriano J, Vallo A. Acute and chronic effects of cisplatin therapy on renal magnesium homeostasis. Med Pediatr Oncol? 1997;28:35-40

2. Lajer $\mathrm{H}$, Kristensen $\mathrm{M}$, Hansen $\mathrm{HH}$, et al. Magnesium depletion enhances cisplatin-induced nephrotoxicity. Cancer Chemother Pharmacol. 2005;56:535-42.

3. Lager H, Daugaard G. Cisplatin and hypomagnesemia. Cancer Treat Rev. 1999;25:47-58.

4. Pabla N, Dong Z. Cisplatin nephrotoxicity: mechanism and renoprotec tive strategies. Kidney Int. 2008;73:994-1007.

5. Milionis HJ, Alexandrides GE, Liberopoulus EN, et al. Hypomagnesemia and concurrent acid-base and electrolyte abnormalities in patients with congestive heart failure. Eur J Heart Fail. 2002;4:167-73.

6. Yamamoto M, Yamaguchi T. Causes and treatment of hypomagnesemia Clin Calcium. 2007;17:1241-8.

7. Hunter RJ, Pace MB, Burns KA, et al. Evaluation of intervention to prevent hypomagnesemia in cervical cancer patients receiving combination cisplatin and radiation treatment. Support Care Cancer: 2009;17:1195-201.

8. Evans TRJ, Harper CL. A randomised study to determine whether routine intravenous magnesium supplements are necessary in patients receiving cisplatin chemotherapy with continuous infusion 5 fluorouracil. Eur J Cancer. 1995;31:174-8.

9. Zarif Yeganeh $M$, Vakili $M$, Shahriari-Ahmadi A, et al. Effect of orat magnesium oxide supplementation on cisplatin-induced hypomagnesemia in cancer patients: a randomized controlled trial. Iran J Public Health. 2016;45:54-62.

10. Yamamoto Y, Watanabe K, Tsukiyama I, et al. Nephroprotective effects of hydration with magnesium in patients with cervical cancer receiving cisplatin. Anticancer Res. 2015;35:2199-204.

11. Kidera $Y$, Kawakami $H$, Sakiyawa $T$, et al. Risk factors for cisplatin-induced nephrotoxicity and potential of magnesium supplementation for renal protection. PLos One. 2014;9:e101902. 